Editorial

Z Herz- Thorax- Gefäßchir 2021 · 35:125 https://doi.org/10.1007/s00398-021-00438-x Angenommen: 3. Mai 2021

(c) Springer Medizin Verlag $\mathrm{GmbH}$, ein Teil von Springer Nature 2021

Liebe Leserinnen und Leser,

eine schleichende, weitgehend gewollte Veränderung durchlebt die Zeitschrift für Herz-, Thorax- und Gefäßchirurgie:

Zunächst war sie unter Roland Hetzer über 20 Jahre deutschsprachige Plattform für überwiegend Originalpublikationen aus unserem Fachgebiet. Mit Einführung des neuen Konzeptes der Herausgeberzeitschrift und Übernahme der Zeitschrift durch die Deutsche Gesellschaft für Thorax-, Herz- und Gefäßchirurgie (2008) änderte sich der Auftrag, in den verschiedenen Rubriken wurden Themen rund um die Herz-, Thoraxund Gefäßchirurgie aufgearbeitet; Originalien wurden selten, Übersichtsarbeiten dominierten. Relativ zügig wurde die Zeitschrift die offizielle Weiterbildungsplattform der DGTHG, neue Rubriken entstanden, das Themenspektrum wurde breiter, das Konzept eines „,bunten Straußes" unterschiedlicher Beiträge rund um unser Fachgebiet wurde realisiert. Dem Ansinnen des Springer-Verlages, Ausgaben mit Themenschwerpunkten zu produzieren, wurde erfolgreich durch das Herausgebergremium und die Schriftführung widerstanden: Wir wollten uns abheben von anderen Zeitschriften, etwas bunter, etwas breiter aufgestellt sein, auch über den Tellerrand einer Fachzeitschrift hinausschauen (tatsächlich gab es viele Jahre eine Rubrik mit dem Namen „Über den Tellerrand", die dann in der dem Jungen Forum der DGTHG zugeordneten Rubrik "\#Leben" aufging - lesen Sie in dieser Ausgabe den Beitrag „Punks,

Um unsere Autoren vor einer missbräuchlichen Verwendung digital verfügbarer Autorensignets zu schützen, werden in der Zeitschrift für Herz-, Thorax- und Gefäßchirurgie künftig keine Autorensignets mehr abgebildet.

Klaus Kallenbach

INCCI HaerzZenter, Luxembourg, Luxemburg

\title{
Redaktioneller Zeitgeist
}

,Pumpe und Plakate"). Für die Zunahme der Wahrnehmung der Zeitschrift war die Entscheidung des Vorstandes der DGTHG, ab Herbst 2013 alle Mitglieder der DGTHG mit der Zeitschrift zu versorgen, richtungweisend. Parallel zu den sich ändernden Lesegewohnheiten, insbesondere der jüngeren Generation von Lesern, passte der Springer-Verlag die digitale Präsentation der Zeitschrift an, die Visibilität nahm dadurch nochmal deutlich zu (Zunahme der Downloads von 15.031 [2010] auf heute 61.798 [2020]).

\section{》) Wir wollten über den Tellerrand einer Fachzeitschrift hinausschauen}

Mit dieser deutlich vergrößerten Wahrnehmung wuchs auch das Interesse der DGTHG an der Zeitschrift: Die „Blauen Seiten", Mitteilungen der DGTHG und bekannt aus dem Thoracic and Cardiovascular Surgeon, werden nun auch in der Zeitschrift für Herz-, Thorax- und Gefäßchirurgie veröffentlicht (s. in dieser Ausgabe „Mitteilungen der DGTHG“). Den oben beschriebenen schleichenden Veränderungsprozess kann man exemplarisch in dieser Ausgabe erkennen: berufspolitische Themen, wie hier die Forderung nach der Etablierung eines Berufsbildes des Kardiotechnikers, werden in einem Konsensuspapier verschiedener medizinischer Fachgesellschaften und in einem Kommentar des damaligen Präsidenten der DGTHG adressiert. Ein weiteres Konsensuspapier zum Ausbildungsmodul „Extrakorporaler Life Support (ECLS)“ wird von Michels et al. zeitgleich in der Zeitschrift für Herz-, Thorax- und Gefäßchirurgie und in den deutschsprachigen Zeit- schriften der jeweiligen Fachgesellschaften publiziert. In den letzten Jahren werden vermehrt Konsensuspapiere in dieser Zeitschrift und zeitgleich anderswo publiziert und kommentiert, was sicherlich der modernen Arbeitsweise medizinischer Fachgesellschaften, auch berufspolitisch, entspricht.

Diese schleichende Veränderung ist zu begrüßen, denn sie macht unsere Zeitschrift noch aktueller. Dazu gehört auch die Rubrik „Im Brennpunkt“, in der schon seit einigen Jahren aktuelle relevante Publikationen zusammengefasst werden. Gleichzeitig bestehen die gewohnten Formate weiter, und ganz im Gegensatz zu unserem Bestreben, eine moderne Zeitschrift mit, dem Zeitgeist folgend, notwendigen Anpassungen zu produzieren, wird in der Rubrik „,historische Profile" zurückgeschaut, auf die Wurzeln unseres Fachgebietes, die man kennen sollte: Der Beitrag von Herrn Ulmer über Francis M. Fontan ist sehr lesenswert!

Ihnen, liebe Leserinnen und Leser, wünsche ich viel Spaß beim Lesen dieser Ausgabe der Zeitschrift für Herz-, Thorax- und Gefäßchirurgie.

Ihr

Prof. Dr. med. Klaus Kallenbach

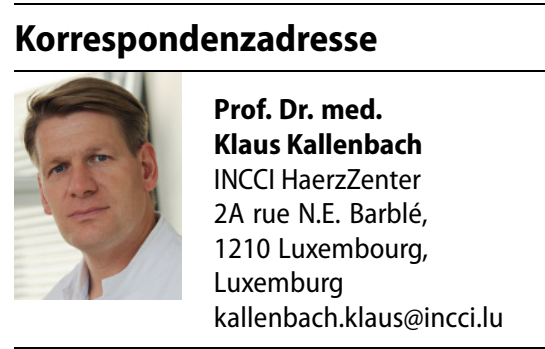

Interessenkonflikt. K. Kallenbach gibt an, dass kein Interessenkonflikt besteht. 